\title{
Screening of Different Bt And Non Bt Cotton Hybrids Against Leaf Hopper, Amrasca Biguttula Biguttula and Thrips. Thrips Tabaci on Bt Cotton
}

\author{
Naveena JB* and Sharanabasappa \\ Department of Entomology, College of Agriculture, Shivamogga, Karnataka, India \\ *Corresponding Author: Naveena JB, Department of Entomology, College of Agriculture, Shivamogga, Karnataka, India.
}

Received: October 17, 2019; Published: October 29, 2019

DOI: $10.31080 / A S A G .2019 .03 .0700$

\begin{abstract}
The field experiment was carried out at Agricultural and Horticultural Research Station, Honnavile farm Shivamogga during Kharif 2016-17. Totally 15 Bt cotton hybrids and their counter non Bt cotton hybrids were screened against sucking pests of cotton like leafhoppers and thrips under unprotected conditions. The mean population of leafhopper across the different Bt cotton hybrids DCH-32 was significantly superior by recording lowest population of 2.99 leafhoppers/3 leaves followed double Bt, Ankur HB-2110 non Bt, Minerva BG-I, SP904 BG-II non Bt, chamundi BG-II non Bt Minerva-I non Bt, double Bt non Bt, MRC-7918, Ankur HB-2110, SP904 BGII and Chamundi BG-II On the contrary, the population of leafhopper was significantly high in MRC 7351 (6.41 leafhoppers/3 leaves) and Ankur HB-2110 (6.22 leafhoppers/3 leaves). However lowest population of thrips were recorded in DCH-32 (4.61 thrips/3 leaves) followed by other Bt cotton hybrids.
\end{abstract}

Keywords: Leafhoppers; Thrips; Bt Cotton; DCH-32; MRC-7351

\section{Introduction}

Insects have been dominating as the most successful forms of life on earth surviving extremes of climatic conditions and have challenged man's effort to eradicate them. Insects have great balancing role in the life process of earth and excel all other animals in both diversity and magnitude. Man's desire to shift nature's balance towards his survival has led to efforts, Cotton (Gossypium spp.) which is a friendly fiber being grown in 111 countries worldwide. In India it is cultivated in $\mathbf{1 1 . 0 0}$ million ha with a production of 33.50 million bales of seed cotton and productivity of $518 \mathrm{~kg} / \mathrm{ha}$ Large area under rain fed situations and extensive replacement of conventional varieties with superior hybrids made the crop easily vulnerable to insect pests. The major reason for the low productivity in cotton is damage caused by insect pests. In India, as many as 162 species of insect-pests are known to attack cotton from sowing to maturity which cause up to 50-60\% loss reported by kranthi. Cotton pests can be primarily divided into bollworms and suck- ing pests. Among sucking pests, aphid Aphisgossypii (Glover), leafhoppers Amrasca biguttula biguttula (Ishida), thrips Thrips tabaci (Lind) and whiteflies Bemisia tabaci (Genn.) are of major importance. These sucking pests occur at all the stages of crop growth and responsible for indirect yield losses. A reduction of 22.85 percent in seed cotton yield due to sucking pests has been reported by satpute. Keeping in the view of this point the main aim of this objective is to study the reaction of Bt cotton hybrids against leafhoppers and thrips of Bt cotton

\section{Material and Methods}

The field experiment was carried out at Agricultural and Horticultural Research Station (AHRS), Honnavile farm Shivamogga during Kharif 2016-17 for screening of Bt cotton hybrids for their reaction to sucking pest. Totally 15 Bt cotton hybrids and their counter non Bt cotton hybrids were screened against sucking pests under unprotected conditions. The hybrids were sown in two rows of 15 m length with a spacing of $90 \mathrm{~cm} \times 60 \mathrm{~cm}$ with two replications. 
The crop was raised following all the recommended agronomic practice except the plant protection measures. Observations on sucking pests viz., thrips and leafhoppers were recorded at monthly intervals starting from 15 days after the sowing continued throughout the crop growth on ten randomly selected plants avoiding the border rows. Sucking pests were recorded on three leaves per plant by selecting top, middle and bottom leaves. The data collected on the population of sucking pests were subjected to ANOVA test by following with $\sqrt{\mathrm{x}}+0.5$ transformations.

\section{Results and Discussion}

The results on population of leafhoppers on different Bt cotton hybrids are furnished in the Table 1. The mean population of leafhopper across the different Bt cotton hybrids DCH-32 was significantly superior by recording lowest population of 2.99 leafhoppers/3 leaves followed by double Bt (3.00 leafhoppers/3 leaves), Ankur HB-2110 non Bt (3.04 leafhoppers/3 leaves), Minerva BG-I (3.39 leafhoppers/3 leaves), SP904 BG-II non Bt (3.51 leafhoppers/3 leaves), Chamundi BG-II non Bt (3.97 leafhoppers/3 leaves) and these Bt cotton hybrids were on par with each other. The next best Bt cotton hybrids were Minerva-I non Bt (4.11 leafhoppers/3 leaves) followed by double Bt non Bt (5.36 leafhoppers/3 leaves), MRC-7918 (5.58 leafhoppers/3 leaves), Ankur HB-2110 (6.22 leafhoppers/3 leaves), SP904 BG-II (5.63 leafhoppers/3 leaves) and Chamundi BG-II (5.85 leafhoppers/3 leaves) where all this Bt cotton hybrids were on par with each other. On the contrary, the population of leafhopper was significantly high in MRC 7351 (6.41 leafhoppers/3 leaves) and Ankur HB-2110 (6.22 leafhoppers/3 leaves). However the incidence of thrips on different Bt cotton hybrids are furnished in the Table 1 . The lowest population of thrips was recorded in DCH-32 (4.61 thrips/3 leaves). On other hand the next best Bt cotton hybrids in the order of superiority were double Bt (10.65 thrips/3 leaves) followed by Ankur HB-2110 non Bt (12.51 thrips/3 leaves), SP904 BG-II non Bt (14.85 thrips/3 leaves) and Minerva BG-I (15.26 thrips/3 leaves) and these hybrids were statistically on par with each other. However the next Bt cotton hybrids in the order of superiority were Ankur HB-2110 (16.27thrips/3 laves) followed by SP904 BG-II (16.61 thrips/3 leaves), double Bt non Bt (17.37 thrips/3 leaves), MRC-7351 (17.62 thrips/3 leaves) and MRC-7918 (17.95 thrips/3 leaves) and these hybrids were statistically on par with each other. On other hand highest population of thrips was recorded on Chamundi BG-II (20.22 thrips/3 leaves) followed by Minerva BG-I non Bt(19.35 thrips/3 leaves) and MRC-7918 non Bt (18.55 thrips/3 leaves), respectively and were statistically on par with each other [1-4]

\begin{tabular}{|c|c|c|c|c|c|c|c|c|c|c|c|c|c|}
\hline \multirow{2}{*}{\multicolumn{2}{|c|}{$\begin{array}{c}\text { Treatment } \\
\text { detail }\end{array}$}} & \multicolumn{5}{|c|}{ No. of A.biguttulabiguttula / 3 leaves } & \multirow{3}{*}{$\begin{array}{c}\text { Mean } \\
5.58\end{array}$} & \multicolumn{5}{|c|}{ No. of Thrips tabaci / 3 leaves } & \multirow{3}{*}{$\begin{array}{c}\text { Mean } \\
17.95 \\
(3.93)^{\mathrm{bc}}\end{array}$} \\
\hline & & \multirow{2}{*}{$\begin{array}{c}\text { August } \\
5.70 \\
(2.43)^{\mathrm{bcd}}\end{array}$} & \multirow{2}{*}{$\begin{array}{c}\begin{array}{c}\text { Septem- } \\
\text { ber }\end{array} \\
4.30 \\
(2.19)^{\operatorname{def}}\end{array}$} & \multirow{2}{*}{$\begin{array}{c}\begin{array}{c}\text { Octo- } \\
\text { ber }\end{array} \\
5.20 \\
(2.39)^{\text {cde }}\end{array}$} & \multirow{2}{*}{$\begin{array}{c}\begin{array}{c}\text { Novem- } \\
\text { ber }\end{array} \\
8.15 \\
(2.94)^{\mathrm{a}} \\
\end{array}$} & \multirow{2}{*}{$\begin{array}{c}\begin{array}{c}\text { Decem- } \\
\text { ber }\end{array} \\
4.55 \\
(2.2)^{\mathrm{bcd}} \\
\end{array}$} & & \multirow{2}{*}{$\begin{array}{c}\text { August } \\
28.05 \\
(5.34)^{\mathrm{a}} \\
\end{array}$} & \multirow{2}{*}{$\begin{array}{c}\begin{array}{c}\text { Septem- } \\
\text { ber }\end{array} \\
28.15 \\
(5.35)^{\mathrm{ab}}\end{array}$} & \multirow{2}{*}{$\begin{array}{c}\begin{array}{c}\text { Octo- } \\
\text { ber }\end{array} \\
21.70 \\
(4.71)^{\mathrm{abc}} \\
\end{array}$} & \multirow{2}{*}{$\begin{array}{c}\begin{array}{c}\text { Novem- } \\
\text { ber }\end{array} \\
11.85 \\
(3.51)^{\text {abcd }} \\
\end{array}$} & \multirow{2}{*}{$\begin{array}{c}\begin{array}{c}\text { Decem- } \\
\text { ber }\end{array} \\
0.00 \\
(0.71)^{\mathrm{d}}\end{array}$} & \\
\hline $\mathrm{T}_{1}$ & $\begin{array}{l}\text { MRC- } \\
7918\end{array}$ & & & & & & & & & & & & \\
\hline $\mathrm{T}_{2}$ & $\begin{array}{l}\text { MRC- } \\
7918, \\
\text { non Bt }\end{array}$ & $\begin{array}{c}3.05 \\
(1.88)^{\mathrm{ef}}\end{array}$ & $\begin{array}{c}5.80 \\
(2.47)^{\mathrm{bcd}}\end{array}$ & $\begin{array}{c}5.35 \\
(2.42)^{\text {cde }}\end{array}$ & $\begin{array}{c}6.00 \\
(2.55)^{\mathrm{de}}\end{array}$ & $\begin{array}{c}3.75 \\
(2.05)^{\mathrm{def}}\end{array}$ & $\begin{array}{c}4.79 \\
(2.28)^{\text {def }}\end{array}$ & $\begin{array}{c}21.05 \\
(4.64)^{\text {de }}\end{array}$ & $\begin{array}{r}28.55 \\
(5.34)\end{array}$ & $\begin{array}{c}27.25 \\
(5.27)^{\mathrm{a}}\end{array}$ & $\begin{array}{c}18.45 \\
(4.35)^{\mathrm{ab}}\end{array}$ & $\begin{array}{c}1.25 \\
(1.32)^{\mathrm{bc}}\end{array}$ & $\begin{array}{c}18.55 \\
(4.11)^{\mathrm{a}}\end{array}$ \\
\hline $\mathrm{T}_{3}$ & MRC- & $\begin{array}{c}9.05 \\
(3.09)^{\mathrm{a}}\end{array}$ & $\begin{array}{c}5.10 \\
(2.36)^{\text {cde }}\end{array}$ & $\begin{array}{c}6.00 \\
(2.55)^{\mathrm{a}} \\
\end{array}$ & $\begin{array}{c}8.00 \\
(2.92)^{\mathrm{a}} \\
\end{array}$ & $\begin{array}{c}3.90 \\
(2.07)^{\mathrm{def}}\end{array}$ & $\begin{array}{r}6.41 \\
(2.6)^{\mathrm{a}}\end{array}$ & $\begin{array}{c}26.50 \\
(5.19)^{\mathrm{ab}}\end{array}$ & $\begin{array}{c}30.00 \\
(5.52)^{\mathrm{ab}}\end{array}$ & $\begin{array}{c}20.05 \\
(4.53)^{\mathrm{bc}}\end{array}$ & $\begin{array}{c}11.55 \\
(3.47)^{\mathrm{bcd}}\end{array}$ & $\begin{array}{c}0.00 \\
(0.71)^{d}\end{array}$ & $\begin{array}{r}17.62 \\
(3.89)^{\mathrm{bc}}\end{array}$ \\
\hline $\mathrm{T}_{4}$ & $\begin{array}{c}\text { MRC- } \\
\text { 7351,non } \\
\text { B t }\end{array}$ & $\begin{array}{c}0.21 \\
(0.84)^{g}\end{array}$ & $\begin{array}{c}8.75 \\
(3.04)^{b c}\end{array}$ & $\begin{array}{c}4.20 \\
(2.16)^{\mathrm{def}}\end{array}$ & $\begin{array}{c}5.50 \\
(2.45)^{\text {cde }}\end{array}$ & $\begin{array}{c}3.55 \\
(2.00)^{\mathrm{def}}\end{array}$ & $\begin{array}{c}4.34 \\
(2.08)^{\text {def }}\end{array}$ & $\begin{array}{c}19.65 \\
(4.49)^{\mathrm{e}}\end{array}$ & $\begin{array}{c}34.20 \\
(5.89)^{\mathrm{ab}}\end{array}$ & $\begin{array}{c}22.25 \\
(4.74)^{\mathrm{abc}}\end{array}$ & $\begin{array}{c}7.50 \\
(2.82)^{\mathrm{de}}\end{array}$ & $\begin{array}{c}0.70 \\
(1.09)^{\mathrm{cd}}\end{array}$ & $\begin{array}{c}16.86 \\
(3.81)^{\mathrm{bc}}\end{array}$ \\
\hline $\mathrm{T}_{5}$ & $\begin{array}{l}\text { Ankur- } \\
\text { HB } 2110\end{array}$ & $\begin{array}{c}6.60 \\
(2.65)^{\text {cde }}\end{array}$ & $\begin{array}{c}4.00 \\
(2.12)^{\mathrm{def}}\end{array}$ & $\begin{array}{c}4.30 \\
(2.18)^{\text {def }}\end{array}$ & $\begin{array}{c}7.85 \\
(2.89)^{\mathrm{ab}}\end{array}$ & $\begin{array}{c}8.30 \\
(2.96)^{a}\end{array}$ & $\begin{array}{c}6.22 \\
(2.57)^{\mathrm{ab}}\end{array}$ & $\begin{array}{c}25.25 \\
(5.07)^{\mathrm{abc}}\end{array}$ & $\begin{array}{l}24.75 \\
5.01)^{\mathrm{b}}\end{array}$ & $\begin{array}{c}18.53 \\
(4.35)^{c}\end{array}$ & $\begin{array}{c}12.30 \\
(3.55)^{\mathrm{abc}}\end{array}$ & $\begin{array}{c}0.00 \\
(0.71)^{d}\end{array}$ & $\begin{array}{c}16.27 \\
(3.76) \\
\text { abc }\end{array}$ \\
\hline
\end{tabular}




\begin{tabular}{|c|c|c|c|c|c|c|c|c|c|c|c|c|c|}
\hline$r_{6}$ & $\begin{array}{c}\text { Ankur- } \\
\text { HB } \\
2110 \text {,non } \\
\text { Bt }\end{array}$ & $\begin{array}{c}3.96 \\
(2.10)^{\text {def }}\end{array}$ & $\begin{array}{c}0.65 \\
(1.07)^{\mathrm{g}}\end{array}$ & $\begin{array}{c}2.40 \\
(1.57)^{\text {ef }}\end{array}$ & $\begin{array}{c}5.40 \\
(2.43)^{\text {cde }}\end{array}$ & $\begin{array}{c}2.80 \\
(1.81)^{\mathrm{ef}}\end{array}$ & $\begin{array}{c}3.04 \\
(1.83)^{\mathrm{def}}\end{array}$ & $\begin{array}{c}3.75 \\
(2.06)^{\mathrm{g}}\end{array}$ & $\begin{array}{c}10.90 \\
(3.37)^{\mathrm{d}}\end{array}$ & $\begin{array}{l}27.25 \\
(5.27)^{\mathrm{a}}\end{array}$ & $\begin{array}{c}11.30 \\
(3.44)^{\text {bcde }}\end{array}$ & $\begin{array}{c}9.85 \\
(3.22)^{\mathrm{a}}\end{array}$ & $\begin{array}{c}12.51 \\
(3.46)^{\mathrm{cd}}\end{array}$ \\
\hline $\mathrm{T}_{7}$ & $\begin{array}{c}\text { Minerva } \\
\text { BG-1 }\end{array}$ & $\begin{array}{c}0.30 \\
(0.89)^{\mathrm{g}}\end{array}$ & $\begin{array}{c}5.30 \\
(2.40)^{\text {cde }}\end{array}$ & $\begin{array}{c}4.40 \\
(2.21)^{\text {def }}\end{array}$ & $\begin{array}{c}4.00 \\
(1.86)^{\mathrm{ef}}\end{array}$ & $\begin{array}{c}2.95 \\
(1.85)^{\mathrm{f}}\end{array}$ & $\begin{array}{c}3.39 \\
(1.9)^{\mathrm{ef}}\end{array}$ & $\begin{array}{c}24.50 \\
(5.00)^{\mathrm{abcd}}\end{array}$ & $(5.07)^{\mathrm{ab}}$ & $\begin{array}{l}18.40 \\
(4.34)^{\mathrm{c}}\end{array}$ & $\begin{array}{c}8.10 \\
(2.93)^{\text {cde }}\end{array}$ & $\begin{array}{c}0.00 \\
(0.71)^{\mathrm{d}}\end{array}$ & $\begin{array}{l}15.26 \\
(3.61)^{\mathrm{bc}}\end{array}$ \\
\hline $\mathrm{T}_{8}$ & $\begin{array}{c}\text { Minerva } \\
\text { BG-1, } \\
\text { non Bt }\end{array}$ & $\begin{array}{c}3.90 \\
(2.09)^{\mathrm{def}}\end{array}$ & $\begin{array}{c}3.75 \\
(2.06)^{\text {def }}\end{array}$ & $\begin{array}{c}5.00 \\
(2.31)^{\text {cde }}\end{array}$ & $\begin{array}{c}5.50 \\
(2.45)^{\text {cde }}\end{array}$ & $\begin{array}{c}2.40 \\
(1.69)^{\mathrm{ef}}\end{array}$ & $\begin{array}{c}4.11 \\
(2.13)^{\text {cde }}\end{array}$ & $\begin{array}{c}23.10 \\
(4.86)^{\text {bcde }}\end{array}$ & $\begin{array}{c}33.10 \\
(5.79)^{\mathrm{ab}}\end{array}$ & $\begin{array}{c}19.80 \\
(4.5)^{\mathrm{bc}}\end{array}$ & $\begin{array}{c}10.75 \\
(3.35)^{\text {bcde }}\end{array}$ & $\begin{array}{c}9.50 \\
(3.13)^{\mathrm{a}}\end{array}$ & $5)^{\mathrm{a}}$ \\
\hline $\mathrm{T}_{9}$ & $\begin{array}{c}\text { Double } \\
\text { Bt }\end{array}$ & $\begin{array}{c}3.52 \\
(2.00)^{\text {def }}\end{array}$ & $\begin{array}{c}2.85 \\
(1.79)^{\mathrm{ef}}\end{array}$ & $\begin{array}{c}2.95 \\
(1.74)^{\mathrm{ef}}\end{array}$ & $\begin{array}{c}3.35 \\
(1.74)^{\mathrm{ef}}\end{array}$ & $\begin{array}{c}2.35 \\
(1.52)^{\mathrm{f}}\end{array}$ & $\begin{array}{c}3.00 \\
(1.87)^{\text {ef }}\end{array}$ & $\begin{array}{l}13.00 \\
(3.67)^{\mathrm{f}}\end{array}$ & $\begin{array}{c}13.28 \\
(3.71)^{\mathrm{cd}}\end{array}$ & $\begin{array}{c}19.90 \\
(4.52)^{\mathrm{bc}}\end{array}$ & $\begin{array}{c}7.05 \\
(2.72)^{\mathrm{e}} \\
\end{array}$ & $\begin{array}{c}0.00 \\
(0.71)^{\mathrm{d}}\end{array}$ & $\begin{array}{c}10.65 \\
(3.07)^{\mathrm{cd}}\end{array}$ \\
\hline $\mathrm{T}_{10}$ & $\begin{array}{l}\text { Dot } \\
\text { Bt,n }\end{array}$ & $\begin{array}{c}4.20 \\
(2.16)^{\mathrm{def}}\end{array}$ & $\begin{array}{c}3.60 \\
(2.02)^{\mathrm{def}}\end{array}$ & $\begin{array}{c}5.50 \\
(2.44)^{\text {cde }}\end{array}$ & $\begin{array}{c}4.60 \\
(2.25)^{\mathrm{ef}}\end{array}$ & $\begin{array}{c}3.20 \\
(1.92)^{\mathrm{ef}}\end{array}$ & $\begin{array}{c}5.36 \\
(2.39)^{\text {cde }}\end{array}$ & $\begin{array}{c}22.00 \\
(4.74)^{\text {cde }}\end{array}$ & $\begin{array}{r}30.50 \\
(5.55)^{\mathrm{ab}}\end{array}$ & $\begin{array}{c}19.30 \\
(4.45)^{\mathrm{bc}}\end{array}$ & $\begin{array}{c}12.20 \\
(3.56)^{\mathrm{abcd}}\end{array}$ & $\begin{array}{c}1.60 \\
(1.44)^{\mathrm{b}}\end{array}$ & $\begin{array}{c}17.37 \\
(4.03)^{\mathrm{ab}}\end{array}$ \\
\hline $\mathrm{T}_{11}$ & $\begin{array}{c}\text { SP } \\
904 \mathrm{BG}-\mathrm{II}\end{array}$ & $\begin{array}{c}5.65 \\
(2.47)^{\text {cde }}\end{array}$ & $\begin{array}{c}9.35 \\
(3.13)^{\mathrm{ab}}\end{array}$ & $\begin{array}{c}4.35 \\
(2.19)^{\text {def }}\end{array}$ & $\begin{array}{c}6.45 \\
(2.64)^{\mathrm{abc}}\end{array}$ & $\begin{array}{c}3.25 \\
(1.94)^{\text {def }}\end{array}$ & $\begin{array}{c}5.63 \\
(2.43)^{\mathrm{abc}}\end{array}$ & $\begin{array}{c}22.87 \\
(4.83)^{\text {bcde }}\end{array}$ & $\begin{array}{c}38.15 \\
(6.21)^{\mathrm{a}}\end{array}$ & $\begin{array}{c}8.23 \\
(2.93)^{\mathrm{d}}\end{array}$ & $\begin{array}{c}18.35 \\
(4.33)^{\mathrm{ab}}\end{array}$ & $\begin{array}{c}0.00 \\
(0.71)^{\mathrm{d}}\end{array}$ & $\begin{array}{l}16.61 \\
(3.7)^{\mathrm{bc}}\end{array}$ \\
\hline${ }_{12}$ & $\begin{array}{c}\text { SP } \\
904 \mathrm{BG}-\mathrm{II} \\
\text {,non Bt }\end{array}$ & $\begin{array}{c}3.80 \\
(2.07)^{\text {def }}\end{array}$ & $\begin{array}{c}2.50 \\
(1.71)^{\mathrm{f}}\end{array}$ & $\begin{array}{c}4.70 \\
(2.26)^{\text {def }}\end{array}$ & $\begin{array}{c}3.80 \\
(2.07)^{\text {def }}\end{array}$ & $\begin{array}{c}2.75 \\
(1.64)^{\text {ef }}\end{array}$ & $\begin{array}{c}3.51 \\
(1.99)^{\text {def }}\end{array}$ & $\begin{array}{c}19.65 \\
(4.49)^{\mathrm{e}}\end{array}$ & $\begin{array}{c}24.00 \\
(4.82)^{\mathrm{bc}}\end{array}$ & $\begin{array}{c}16.95 \\
(4.16)^{\mathrm{c}}\end{array}$ & $\begin{array}{c}11.45 \\
(3.45)^{\text {bcde }}\end{array}$ & $\begin{array}{c}2.20 \\
(1.61)^{\mathrm{bc}}\end{array}$ & $\begin{array}{c}14.85 \\
(3.74) \\
\text { bcd }\end{array}$ \\
\hline $\mathrm{T}_{13}$ & $\begin{array}{c}\text { Chamun- } \\
\text { di BG-II }\end{array}$ & $\begin{array}{c}3.20 \\
(1.92)^{\mathrm{ef}}\end{array}$ & $\begin{array}{c}9.80 \\
(3.21)^{\mathrm{a}} \\
\end{array}$ & $\begin{array}{c}5.90 \\
(2.53)^{\mathrm{ab}} \\
\end{array}$ & $\begin{array}{c}6.20 \\
(2.59)^{\mathrm{abc}}\end{array}$ & $\begin{array}{c}4.15 \\
(2.14)^{\text {bcd }}\end{array}$ & $\begin{array}{c}5.85 \\
(2.48)^{\mathrm{abc}} \\
\end{array}$ & $\begin{array}{c}22.20 \\
(4.76)^{\text {cde }}\end{array}$ & $\begin{array}{c}34.80 \\
(5.94)^{\mathrm{ab}}\end{array}$ & $\begin{array}{c}25.45 \\
(5.08)^{\mathrm{ab}}\end{array}$ & $\begin{array}{l}20.60 \\
(4.59)^{\mathrm{a}}\end{array}$ & $\begin{array}{c}1.25 \\
(1.32)^{\mathrm{bc}}\end{array}$ & $\begin{array}{c}20.22 \\
(4.27)^{\mathrm{a}}\end{array}$ \\
\hline $\mathrm{T}_{14}$ & $\begin{array}{c}\text { Chamun- } \\
\text { di BG- } \\
\text { II,nonBt }\end{array}$ & $\begin{array}{c}3.95 \\
(2.04)^{\mathrm{def}}\end{array}$ & $\begin{array}{c}3.75 \\
(2.05)^{\mathrm{def}}\end{array}$ & $\begin{array}{c}6.25 \\
(2.6)^{\mathrm{abc}} \\
\end{array}$ & $\begin{array}{c}3.50 \\
(2.00)^{\text {def }}\end{array}$ & $\begin{array}{c}2.40 \\
(1.68)^{\mathrm{ef}}\end{array}$ & $\begin{array}{c}3.97 \\
(2.09)^{\text {def }} \\
\end{array}$ & $\begin{array}{l}20.70 \\
(4.6)^{\mathrm{ef}}\end{array}$ & $\begin{array}{c}33.35 \\
(5.82)^{\mathrm{ab}}\end{array}$ & $\begin{array}{c}16.35 \\
(4.08)^{\mathrm{c}}\end{array}$ & $\begin{array}{c}10.35 \\
(3.29)^{\text {bcde }}\end{array}$ & $\begin{array}{c}1.50 \\
(1.41)^{\mathrm{bc}}\end{array}$ & $\begin{array}{c}16.45 \\
(3.85) \\
\text { bcd }\end{array}$ \\
\hline $\mathrm{T}_{15}$ & DCH-32 & $\begin{array}{c}2.30 \\
(1.66)^{\mathrm{ef}}\end{array}$ & $\begin{array}{c}2.60 \\
(1.75)^{\mathrm{ef}}\end{array}$ & $\begin{array}{c}4.75 \\
(2.29)^{\text {def }}\end{array}$ & $\begin{array}{c}2.30 \\
(1.64)^{\mathrm{ef}}\end{array}$ & $\begin{array}{c}3.60 \\
(1.98)^{\text {def }}\end{array}$ & $\begin{array}{c}2.99 \\
(1.85)^{\text {ef }}\end{array}$ & $\begin{array}{c}2.80 \\
(1.81)^{\mathrm{g}}\end{array}$ & $\begin{array}{c}7.09 \\
(2.69)^{\mathrm{d}}\end{array}$ & $\begin{array}{c}0.63 \\
(1.04)^{\mathrm{e}}\end{array}$ & $\begin{array}{c}7.35 \\
(2.52)^{\text {bcde }}\end{array}$ & $\begin{array}{c}1.35 \\
(1.36)^{\mathrm{bc}}\end{array}$ & $\begin{array}{c}4.61 \\
(2.08) \\
\text { def }\end{array}$ \\
\hline & SEM \pm & 14 & 0.61 & 0.13 & 0.19 & 0.71 & 0.14 & 0.30 & 0.12 & 0.19 & 0.17 & 0.54 & 0.18 \\
\hline & @ $=0.05$ & 0.42 & 1.76 & 0.39 & 0.57 & 2.10 & 0.44 & 0.67 & 0.36 & 0.59 & 0.51 & 1.56 & 0.56 \\
\hline & CV\% & 8.69 & 14.08 & 14.15 & 15.23 & 14.88 & 8.64 & 15.29 & 12.16 & 10.73 & 12.63 & 12.31 & 14.48 \\
\hline
\end{tabular}

Table 1: Screening of different Bt and non Bt cotton hybrids against leafhoppers, Amrasca biguttula biguttula and thrips, Thrips tabaci.

Figures in parenthesis are Öx +0.5 transformed value Means in the columns followed by the same alphabet do not differ significantly by DMRT $(\mathrm{P}=0.05)$ 


\section{Conclusion}

Population of leafhoppers on different Bt cotton hybrids are furnished in the Table 1 . The mean population of leafhopper across the different Bt cotton hybrids DCH-32 was significantly superior by recording lowest population of 2.99 leafhoppers/3 leaves followed by double Bt (3.00 leafhoppers/3 leaves), Ankur HB-2110 non Bt (3.04 leafhoppers/3 leaves), Minerva BG-I (3.39 leafhoppers/3 leaves), SP904 BG-II non Bt (3.51 leafhoppers/3 leaves), Chamundi BG-II non Bt (3.97 leafhoppers/3 leaves) and these Bt cotton hybrids were on par with each other. The next best Bt cotton hybrids were Minerva-I non Bt (4.11 leafhoppers/3 leaves) followed by double Bt non Bt (5.36 leafhoppers/3 leaves), MRC-7918 (5.58 leafhoppers/3 leaves), Ankur HB-2110 (6.22 leafhoppers/3 leaves), SP904 BG-II (5.63 leafhoppers/3 leaves) and Chamundi BG-II (5.85 leafhoppers/3 leaves) where all this Bt cotton hybrids were on par with each other. On the contrary, the population of leafhopper was significantly high in MRC 7351 (6.41 leafhoppers/3 leaves) and Ankur HB-2110 (6.22 leafhoppers/3 leaves) Udikeri and coworkers reported that the results were significant different in respect of the leafhopper populations for Bt and non-Bt cotton, but were not significant with respect to thrips and aphid at Dharwad. The thrips population ranged from 8.10 to 10.80 /leaf. Although all the Bt genotypes had higher thrips populations, they were on a par with the non-Bt genotypes as well as the standard controls (DHH-11 and NHH-44). The aphid population was low in all the treatments including Bt genotypes (6.35 to 8.55/leaf) and there was no significant difference. There was significant variation in the leafhopper populations between the different genotypes and the population was lower (1.30/leaf) in MECH 184 non-Bt which was on par with MECH 184 Bt, MECH 162 Bt and MECH 162 nonBt. However the incidence of thrips on different Bt cotton hybrids are furnished in the Table 1. The lowest population of thrips was recorded in DCH-32 (4.61 thrips/3 leaves). On other hand the next best $\mathrm{Bt}$ cotton hybrids in the order of superiority were double $\mathrm{Bt}$ (10.65 thrips/3 leaves) followed by Ankur HB-2110 non Bt (12.51 thrips/3 leaves), SP904 BG-II non Bt (14.85 thrips/3 leaves) and Minerva BG-I (15.26 thrips/3 leaves) and these hybrids were statistically on par with each other. However the next Bt cotton hybrids in the order of superiority were Ankur HB-2110 (16.27thrips/3 laves) followed by SP904 BG-II (16.61 thrips/3 leaves), double Bt non Bt (17.37 thrips/3 leaves), MRC-7351 (17.62 thrips/3 leaves) and MRC-7918 (17.95 thrips/3 leaves) and these hybrids were sta- tistically on par with each other. On other hand highest population of thrips was recorded on Chamundi BG-II (20.22 thrips/3 leaves) followed by Minerva BG-I non Bt (19.35 thrips/3 leaves) and MRC7918 non Bt (18.55 thrips/3 leaves), respectively and were statistically on par with each other. Probable reasons attributed for higher incidence of thrips might be due to morphological features of genotypes, and prevailing environmental factors which supported in higher pest activity. The present results are in agreement with the findings of many workers. Muhammad and coworkers 2012 reported that AA-802 and VH-259 with 3.40 and 4.45 thrips per leaf were resistant and susceptible genotypes.

\section{Bibliography}

1. Satpute US., et al. "Avoidable field losses due to sucking pests and bollworms in cotton". Journal of Applied Zoological Research 1.2 (1990): 67-72.

2. Kranthi KR and Kranthi NR. "Modelling adaptability of the cotton bollworm, Helicoverpa armigera (Hubner) to Bt cotton in India". Current Science 87 (2004): 1096-1107.

3. Udikeri SS., et al. "Performance of Bt cotton genotypes under unprotected conditions". World Cotton Research Conference-3, Cape Town (2003): 1281-1286.

4. Muhammad Rafiq S., et al. "Comparison of resistance among different cultivars of cotton against thrips tabaci under unsprayed conditions". Pakistan Entomologist 34.1 (2012): 8385.

\section{Volume 3 Issue 11 November 2019 C All rights are reserved by Naveena JB and Sharanabasappa.}

